\title{
Classification and Evaluation of Mobility Metrics for Mobility Model Movement Patterns in Mobile Ad-Hoc Networks
}

\author{
Santosh Kumar S C Sharma Bhupendra Suman \\ Indian Institute of Technology, Roorkee-India \\ amu.santosh@gmail.com, subhash1960@rediffmail.com, \\ bhupendra_suman@rediffmail.com
}

\begin{abstract}
A mobile ad hoc network is collection of self configuring and adaption of wireless link between communicating devices (mobile devices) to form an arbitrary topology and multihop wireless connectivity without the use of existing infrastructure. It requires efficient dynamic routing protocol to determine the routes subsequent to a set of rules that enables two or more devices to communicate with each others. This paper basically classifies and evaluates the mobility metrics into two categories- direct mobility metrics and derived mobility metrics. These two mobility metrics has been used to measure different mobility models, this paper considers some of mobility models i.e Random Waypoint Model, Reference Point Group Mobility Model, Random Direction Mobility Model, Random Walk Mobility Model, Probabilistic Random Walk, Gauss Markov, Column Mobility Model, Nomadic Community Mobility Model and Manhattan Grid Model.
\end{abstract}

\section{KEYWORDS}

Mobility metrics, Mobility Models, Connectivity Graph

\section{Introduction}

Mobile ad hoc network (MANET) is a collection of mobile nodes forming a temporary network, without the aid of pre-establishment network infrastructure, although commercial wireless technologies are generally based on towers and high-power base stations [1,2]. They are fixed in location and relationship to their client devices. Many researchers have shown their interest in the field of MANET and all sorts of protocols aiming at different issues. The performance of these protocols need to be carefully evaluated before they are ready for the commercial market, so for this network simulation plays vital role as well as a key method to comprehend the overall performance of the MANET. The performance of these protocols could be evaluated with the imperative mobility model that precisely represents mobile nodes (MNs) to provide realistic performance measurement. In mobility modelling research, there are two direction of research which could be performed. First direction is towards designing of new models which predicts new era of real world scenario. Second direction is to analyze mobility models on account of mobility metrics and influences of mobility models on routing protocols. There are many mobility models have been proposed in the literature $(3,4,5,6,7,8,9,10,11,12,13,14$, $15,16,17)$. Brief descriptions of some mobility models are: 
International journal on applications of graph theory in wireless ad hoc networks and sensor networks

(GRAPH-HOC) Vol.3, No.3, September 2011

Random Waypoint Model: it includes pause times between changes in direction and speed .A mobile node stay in one location for certain period of pause time [8].

Random Walk Mobility Model: an MN moves from its current location to a new location by randomly choosing a direction and speed in which to travel [10].

Probabilistic Random Walk Model: A model that utilizes a set of probabilities to determine the next position of an MN [11].

Random Direction Mobility Model: A model that forces MNs to travel to the edge of the simulation area before changing direction and speed, to overcome density waves produced by RWP[12].

Reference Point Group Mobility Model: A group mobility model where group movements are based upon the path travelled by a logical center [13].

Gauss Markov: A model that uses one tuning parameter to vary the degree of randomness in the mobility pattern. Initially each MN is assigned a current speed and direction [14].

Column Mobility Model: A group mobility model where the set of MNs form a line and are uniformly moving forward in a particular direction [15].

Nomadic Community Mobility Model: A group mobility model where a set of MNs move together from one location to another $[15,16]$.

Manhattan Grid Model: In this model nodes move only on predefined paths. The arguments -u and -v set the number of blocks between the paths [17].

This paper basically classifies and evaluates the mobility metrics into two categories- direct mobility metrics and derived mobility metrics. These two mobility metrics has been used to measure different mobility models which are tabulated in table 1.

\section{Classification of Mobility Metrics}

The mobility model can be classified based on two kinds of mobility metrics categories, first is direct mobility metrics and second is derived mobility metrics [6]. The direct mobility metrics like host speed or relative speed, are measurement of clear physical behavioural meaning while the derived mobility metrics, like graph connectivity, are measurement of physical observation through mathematical modelling. A good effort of mobility models classification has been carried out based on mobility metrics taking account of above two categories and tabulated in table 1 by several studies of the literature $[3,4,5,6,7,8,9,10,11,12,13,14,15,16,17]$. 
International journal on applications of graph theory in wireless ad hoc networks and sensor networks

(GRAPH-HOC) Vol.3, No.3, September 2011

Table 1 Classification of Mobility Metrics \& its characteristics

\begin{tabular}{|c|c|c|c|c|c|}
\hline Sl.No & $\begin{array}{l}\text { Mobility } \\
\text { Metrics } \\
\text { Categories }\end{array}$ & \multicolumn{2}{|r|}{ Metrics class } & Characteristics & Mobility Model \\
\hline \multirow[t]{3}{*}{1.} & \multirow{3}{*}{$\begin{array}{l}\text { Direct } \\
\text { Mobility }\end{array}$} & \multicolumn{2}{|c|}{ Random based } & $\begin{array}{l}\text { Without any dependencies } \\
\text { and restriction invoked in } \\
\text { model }\end{array}$ & $\begin{array}{l}\text { RWP, RWM, } \\
\text { RDM, Cluster }\end{array}$ \\
\hline & & \multicolumn{2}{|c|}{$\begin{array}{l}\text { Temporal } \\
\text { dependence }\end{array}$} & $\begin{array}{l}\text { A node actual movement } \\
\text { influenced with its past } \\
\text { movement }\end{array}$ & GM, S R \\
\hline & & \multicolumn{2}{|c|}{$\begin{array}{l}\text { Spatial } \\
\text { dependence }\end{array}$} & $\begin{array}{l}\text { The movement of a node } \\
\text { influenced by node around } \\
\text { it happen in group mobility }\end{array}$ & $\begin{array}{lr}\text { RPGM, } & \text { SGM, } \\
\text { CMM, } & \text { NCMM, } \\
\text { Pursue } & \end{array}$ \\
\hline \multirow[t]{3}{*}{2.} & \multirow{3}{*}{$\begin{array}{l}\text { Derived } \\
\text { mobility }\end{array}$} & \multicolumn{2}{|c|}{$\begin{array}{l}\text { Geographical } \\
\text { restrictions }\end{array}$} & $\begin{array}{l}\text { Node movement restricted } \\
\text { in certain geographical } \\
\text { area }\end{array}$ & $\begin{array}{l}\text { Graph Based, } \\
\text { Geographic } \\
\text { division based, } \\
\text { Map-based, } \\
\text { Voronoi-based }\end{array}$ \\
\hline & & & $\begin{array}{l}\text { Hybrid } \\
\text { structure }\end{array}$ & $\begin{array}{l}\text { All mobility metrics } \\
\text { classes are integrated to } \\
\text { attain the structure }\end{array}$ & $\begin{array}{l}\text { CVTM, Freeway, } \\
\text { MGM, }\end{array}$ \\
\hline & & b. & $\begin{array}{l}\text { User - } \\
\text { Oriented-with } \\
\text { three } \\
\text { components }\end{array}$ & $\begin{array}{l}\text { provide an modeling } \\
\text { approach for complex } \\
\text { mobility scenario- spatial } \\
\text { environment, observation } \\
\text { based, temporal and spatial } \\
\text { dependencies }\end{array}$ & $\begin{array}{l}\text { Tactical scenario } \\
\text { Model like- } \\
\text { Catastrophe- } \\
\text { scenario, platoon, } \\
\text { Disaster-area } \\
\text { model }\end{array}$ \\
\hline
\end{tabular}

\subsection{Direct Mobility Metrics}

This mobility metrics is basically a protocol independent metrics, its attempt are to extract the characteristics of mobility behaviour in between the mobile nodes. It is known as direct mobility because of it measures the host movement directly like average host speed. There are some other metrics are defined in this categories like average relative speed, temporal dependence and spatial dependence. An attempt has been carried out to characterize the temporal dependence of the movements of an individual nodes and the spatial dependence between various nodes $[6,18]$

\subsubsection{Relative speed}

It is defined based on relative speed $[6,18]$ of all pairs of nodes in networks over time $t$ i.e. the speed of first node $\mathrm{i}$, relative to the second node $\mathrm{j}$.

$$
R S(i, j, t)=\left|\vec{V}_{i}(t)-\vec{V}_{j}(t)\right|
$$

\section{Average Relative Speed}

Average relative speed RS $(i, j)$ of hosts $i, j$ at time $t$ will be-

$$
\overline{R S}=\frac{\sum_{i=1}^{N} \sum_{j=1}^{N} \sum_{t=1}^{T} R S(i, j, t)}{P}
$$

where $P$ is the number of tuples $(i, j, t)$ such that $\operatorname{RS}(i, j, t) \neq 0$. 
International journal on applications of graph theory in wireless ad hoc networks and sensor networks

(GRAPH-HOC) Vol.3, No.3, September 2011

\subsubsection{Degree of Temporal Dependence}

The temporal dependencies imply how an individual node changes its velocity with respect to time or a node actual movement influenced with its past movement [6]. For each node, it is defined as a product of relative direction and relative speed (relative to its past itself) i.e.

$$
D_{\text {temporal }}\left(i, t, t^{\prime}\right)=R D\left(\vec{v}_{i}(t), \vec{v}_{i}\left(t^{\prime}\right)\right) * S R\left(\vec{v}_{i}(t), \vec{v}_{i}\left(t^{\prime}\right)\right)
$$

The value of $\mathrm{D}_{\text {temporal }}\left(i, t, t^{\prime}\right)$ will increased if the node moves in the same direction and almost at the similar speed and decreases if the relative direction or the speed ratio decreases over a certain time interval. $\mathrm{D}_{\text {spatial }}(i, j, t)$ will be zero if

$$
\left|t-t^{\prime}\right|>c \Rightarrow D_{\text {temporal }}\left(i, t, t^{\prime}\right)=0
$$

where $\mathrm{c}>0$ is a constant

\section{Average Degree of Temporal Dependence}

The average degree of temporal dependence $\mathrm{D}_{\text {temporal }}\left(\mathrm{i}, \mathrm{t}, \mathrm{t}^{\prime}\right)$ is the value of averaged over nodes and time instants [6] i.e

$$
D_{\text {temporal }}=\frac{\sum_{i=1}^{N} \sum_{t=1}^{T} \sum_{t^{\prime}=1}^{T} D_{\text {temporal }}\left(i, j, t^{\prime}\right)}{P}
$$

where $P$ is the number of tuples $\left(i, t, t^{\prime}\right)$ such that $\mathrm{D}_{\text {temporal }}\left(i, t, t^{\prime}\right) \neq 0$. It has two conditions that is obvious, first, if the present velocity of a node is fully independent of its velocity at previous time period, then the mobility pattern is expected to have a smaller value for $\mathrm{D}_{\text {temporal }}$. Second, if the current velocity is strongly dependent on the velocity at some previous time step, then the mobility pattern is expected to have a higher value for $\mathrm{D}_{\text {temporal }}$.

\subsubsection{Degree of Spatial Dependence}

The degree of spatial dependence is a measure of a node's correlation with others nodes in the networks [6]. This could be measured with equ. (6).

$$
D_{\text {spatial }}(i, j, t)=R D\left(\vec{v}_{i}(t), \vec{v}_{j}(t)\right) * S R\left(\vec{v}_{i}(t), \vec{v}_{j}(t)\right)
$$

The value of $\mathrm{D}_{\text {spatial }}(\mathrm{i}, \mathrm{j}, \mathrm{t}$ ) will increases when nodes $i$ and $j$ moves in same direction with almost similar speed and decreases when nodes $i$ and $j$ moves in relative direction or dissimilar speed over certain time interval. The spatially dependent on a far away node will be zero and satisfy the conditions of equ. (7).

$$
D_{i, j}(t)>c * R \Rightarrow D_{\text {spatial }}(i, j, t)=0
$$

where $c>0$ is a constant.

\section{Average Degree of Spatial Dependence}

The average degree of spatial dependence is an average of degree of spatial dependence of all nodes pairs in the network [6] i.e. 
International journal on applications of graph theory in wireless ad hoc networks and sensor networks

(GRAPH-HOC) Vol.3, No.3, September 2011

$$
D_{\text {spatial }}=\frac{\sum_{=1}^{T} \sum_{=1}^{N} \sum_{j=i+1}^{N} D_{\text {spatial }}(i, j, t)}{P}
$$

where $P$ is the number of tuples $(i, j, t)$ such that $\mathrm{D}_{\text {spatial }}(i, j, t) \neq 0$.

\subsection{Derived mobility metrics}

The derived mobility metrics captures the property of graph theoretic models as well as some mathematical models. It is well known that the mobility of nodes dependent over the network topology dynamics, therefore it is oblivious that the performance of routing protocol will also affected by this. To study the effect of mobility it requires analyzing the connectivity graph between the mobile nodes [6].

\section{Connectivity Graph Metrics}

The connectivity graph is the graph $G=(V, E)$ where $|V|=N$, a link $(i, j) \in E$ iff $D_{i, j}(t) \leq R$. Let $X(i, j, t)$ be an indicator random variable which has a value 1 iff there is a link between nodes $i$ and $j$ at time $t[6] . X(i, j)=\max ^{\mathrm{T}}{ }_{\mathrm{t}=1} X(i, j, t)$ be an indicator random variable which is 1 if a link existed between nodes $i$ and $j$ at any time during the simulation, 0 otherwise. The graph connectivity metrics includes total link, number of link changes, link duration and path availability.

\subsubsection{Number of Link Changes}

Number of link changes for a pair of nodes $i$ and $j$ is the number of times the link between them transitions from "down" to "up" [6] and defined as-

$$
L C(i, j)=\sum_{t=1}^{T} C(i, j, t)
$$

Where $\mathrm{C}(\mathrm{i}, \mathrm{j}, \mathrm{t})$ is indicator random variable such that $C(i, j, t)=1$ iff $X(i, j, t-1)=0$ and $X(i, j$, $t)=1$ i.e. if the link between nodes $i$ and $j$ is down at time $t-1$, but comes up at time $t$.

\section{Average Number of Link Changes}

Average Number of Link Changes is the value of $\operatorname{LC}(i, j)$ averaged over node pairs satisfying certain condition [6] i.e

$$
\overline{L C}=\frac{\sum_{i=1}^{N} \sum_{j=i+1}^{N} L C(i, j)}{P}
$$

where $P$ is the number of pairs $i, j$ such that $X(i, j) \neq 0$

\subsubsection{Link Duration}

It is a duration of the link active between two nodes $i$ and $j$. It measures the stability of nodes between these two nodes and defined as [6]- 
International journal on applications of graph theory in wireless ad hoc networks and sensor networks (GRAPH-HOC) Vol.3, No.3, September 2011

$$
L D(i, j)=\left\{\begin{array}{lc}
\frac{\sum_{t=1}^{T} X(i, j, t)}{L C(i, j)} & \text { ifLC }(i, j) \neq 0 \\
\sum_{t=1}^{T} X(i, j, t) & \text { othewise }
\end{array}\right.
$$

\section{Average Link Duration}

It is the value of $\operatorname{LD}(i, j)$ averaged over node pairs meeting certain condition[6]-

$$
\overline{L D}=\frac{\sum_{i=1}^{N} \sum_{j=i+1}^{N} L D(i, j)}{P}
$$

where $P$ is the number of pairs $i, j$ such that $X(i, j) \neq 0$.

\subsubsection{Path Availability}

It is the fraction of time during which a path is available between two nodes $i$ and $j$. At this fraction of time pairs of node communicate traffic with available path [6]. So, the path availability is defined as-

$$
P A(i, j)=\left\{\begin{array}{cc}
\frac{\left.\sum_{t=s t a r t}^{T} A, j\right)}{T-\operatorname{start}(i, j)} & \text { if } T-\operatorname{start}(i, j)>0 \\
0 & \text { otherwise }
\end{array}\right.
$$

where $A(i, j, t)$ is an indicator random variable which has a value 1 if a path is available from node $i$ to node $j$ at time $t$, and has a value 0 otherwise. $\operatorname{Start}(i, j)$ is the time at which the communication traffic between nodes $i$ and $j$ starts.

\section{Average Path Availability}

It is the value of PA $(i, j)$ averaged over node pairs meeting certain condition [6].

$$
\overline{P A}=\frac{\sum_{i=1}^{N} \sum_{j=i+1}^{N} P A(i, j)}{P}
$$

where $P$ is the number of pairs $i, j$ such that $T-\operatorname{start}(i, j)>0$.

\subsubsection{Total links}

Total links prediction value probabilistic factor and predicts the future status of wireless link. The status of link is depends on various system and environmental factors that affect transmitter and receiver range [19]. Nodes are mobile in wireless ad hoc networks therefore, some links will be broken and new links will be established in the networks. The evaluation of total links in transmission range 200 meters is carried out for the some mobility model and shown in figure 7.

\section{Simulation Based Validation of Mobility Metrics}

For the validation of mobility metrics, we used BonnMotion, a Java software which creates and analyses mobility scenarios. It is developed within the Communication Systems group at the 
International journal on applications of graph theory in wireless ad hoc networks and sensor networks

(GRAPH-HOC) Vol.3, No.3, September 2011

Institute of Computer Science of the University of Bonn, Germany, where it serves as a tool for the investigation of mobile ad hoc network characteristics. The scenarios can also be exported for the network simulators ns-2, GloMoSim/QualNet, COOJA, MiXiM and ONE [20]. In our experiment, a Network simulator ns-2 is used for creating and analysing mobility scenario.

\subsection{Scenario description}

In this paper, mobility scenario generator produced different mobility patterns for our considered mobility model i.e Random waypoint model, Reference Point Group Model, Random Direction Model, Random Walk, Probabilistic Random Walk, Gauss Markvo, Column mobility model, and Nomadic Community Mobility Model. In all these mobility patterns, 40 mobile nodes move in the area of $1000 \times 1000 \mathrm{~m}$ for a period of 900 seconds. The simulation is carried out with varying speed of 10,20,30,40,50, $60 \mathrm{~m} / \mathrm{s}$ and fixed transmission range of 200 meters. For Reference point group mobility model, there are two different mobility scenarios. First is a single group of 40 nodes and second is 4 groups of 10 nodes each moving independently with overlapping fashion. Both speed deviation ratio and angle deviation ratio is considered 0.1 .

\subsection{Validation of Direct Mobility Metrics}

\section{Average Relative Speed}

In this paper, from the simulation its has been observed that the average relative speed of Column Mobility Model is lowest as compared to others mobility models, as its nature of group movement pattern, we considered 4 groups in the simulated scenario, however, its average relative speed is less as compared to RPGM (1-groups 40 nodes) and RPGM (4-groups 10 each). The Nomadic Community Mobility Model is also based on group mobility pattern. Each group of mobile nodes has an invisible reference node that they track around the simulation. The average relative speed is good as compared to others i.e Column and RPGM model but not consistent as $\mathrm{V}_{\max }$ increases.

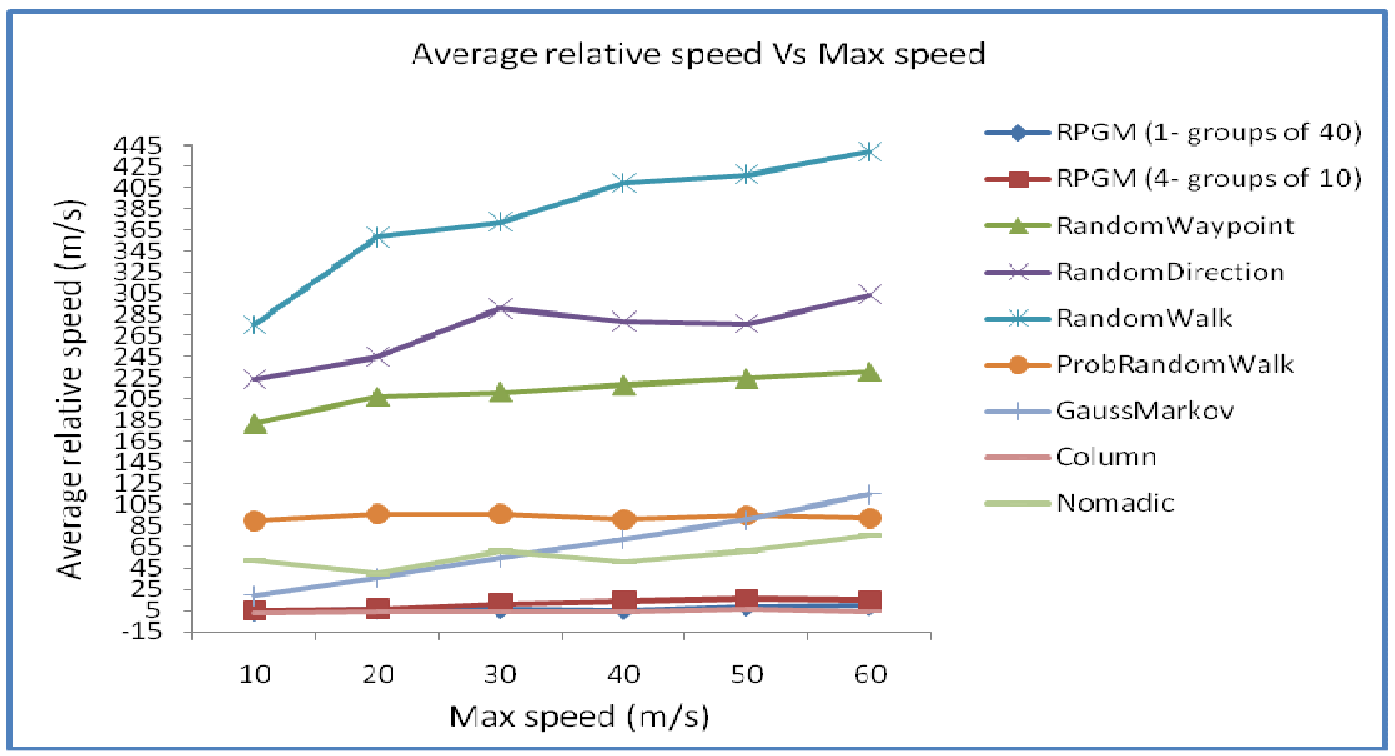

Figure 1 Average relative speed Vs Max speed (m/s) 
International journal on applications of graph theory in wireless ad hoc networks and sensor networks

(GRAPH-HOC) Vol.3, No.3, September 2011

As its simplicity, the average relative speed of all pairs of nodes in Random Walk mobility is highest than any other considered simulated model as the $\mathrm{V}_{\max }$ increases. The average relative speed of all pair of nodes in Random Direction is also increases as the $\mathrm{V}_{\max }$ increases and get highest than rest of other models except Random Walk as shown in figure 1. In Random Waypoint model the average relative speed increases almost linearly as the $\mathrm{V}_{\max }$ increases and achieves highest value rather than others model except Random Walk and Random Direction respectively. In Probabilistic Random walk model, the average relative speed is approximately consistent as shown in figure 1. The average relative speed of all pair's nodes in Gauss Markov Model is linearly increases as the $\mathrm{V}_{\max }$ increases and finally gets highest value except Random Walk, Random Direction and Random Waypoint model.

\section{Average Degree of Temporal Dependence}

This metrics could not differentiate accurately various mobility patterns but still some effort has been made in this paper. Figure 2 shows that none of mobility model signify average temporal dependence with respect to increasing $\mathrm{V}_{\max }$ all has gone in negative value except Probabilistic Random Walk model. In Probabilistic Random Walk model this metrics value increases upto $\mathrm{V}_{\max } 30 \mathrm{~m} / \mathrm{s}$ but gets sudden fall as speed increases more than $30 \mathrm{~m} / \mathrm{s}$.

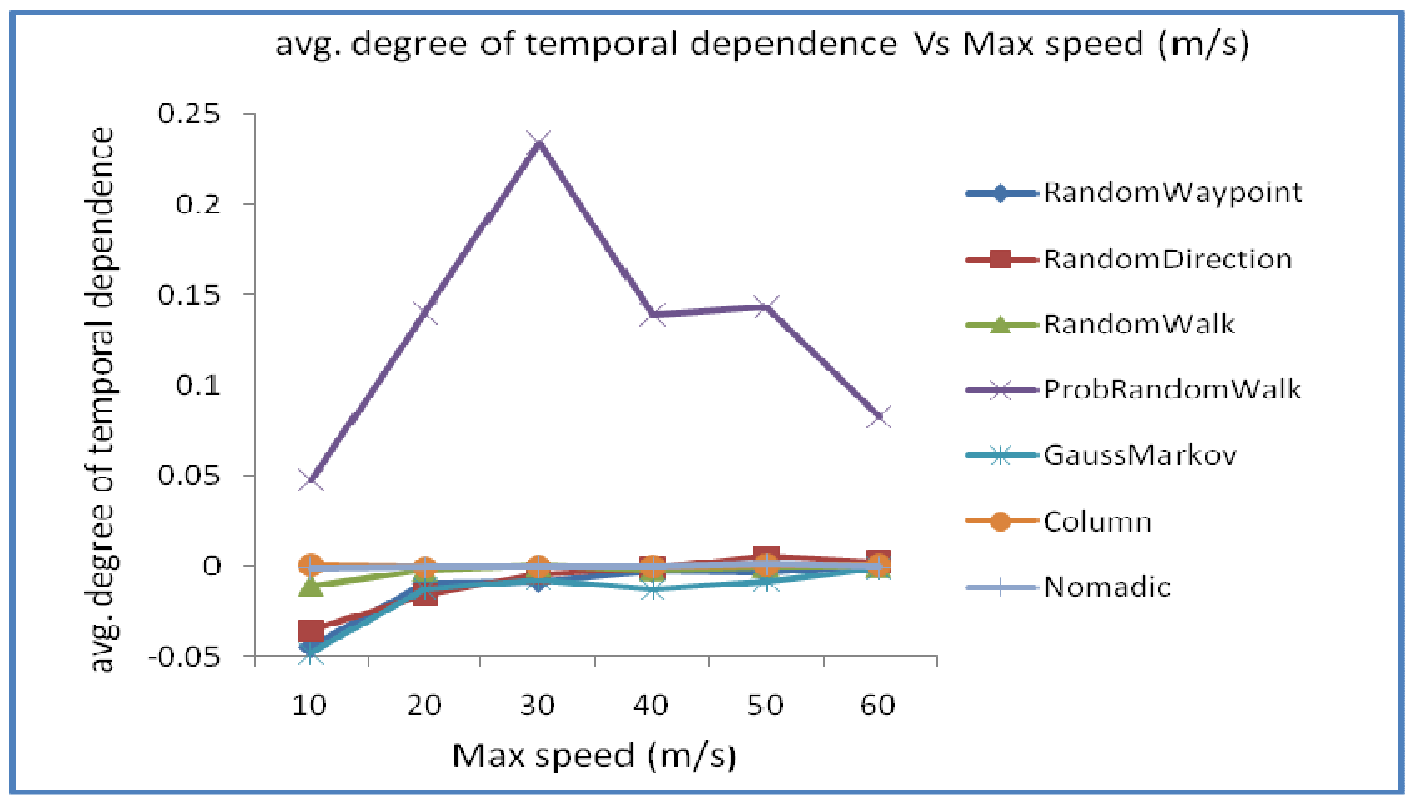

Figure 2 Average degree of temporal dependence Vs Max speed (m/s)

The above results cause uncertainty for validation of different mobility pattern .The usefulness of this metrics is still in research.

\section{Average Degree of Spatial Dependence}

The average degree of spatial dependence is maximum in RPGM (1-group of 40 nodes), RPGM (4- groups of 10 nodes ) and Nomadic model respectively but as the $V_{\max }$ increases then it decreases from its maximum spatial dependencies while in the case of Nomadic Community Mobility Model average degree of spatial dependence increases a little as $\mathrm{V}_{\max }$ increases as shown in figure 2 . 
International journal on applications of graph theory in wireless ad hoc networks and sensor networks

(GRAPH-HOC) Vol.3, No.3, September 2011

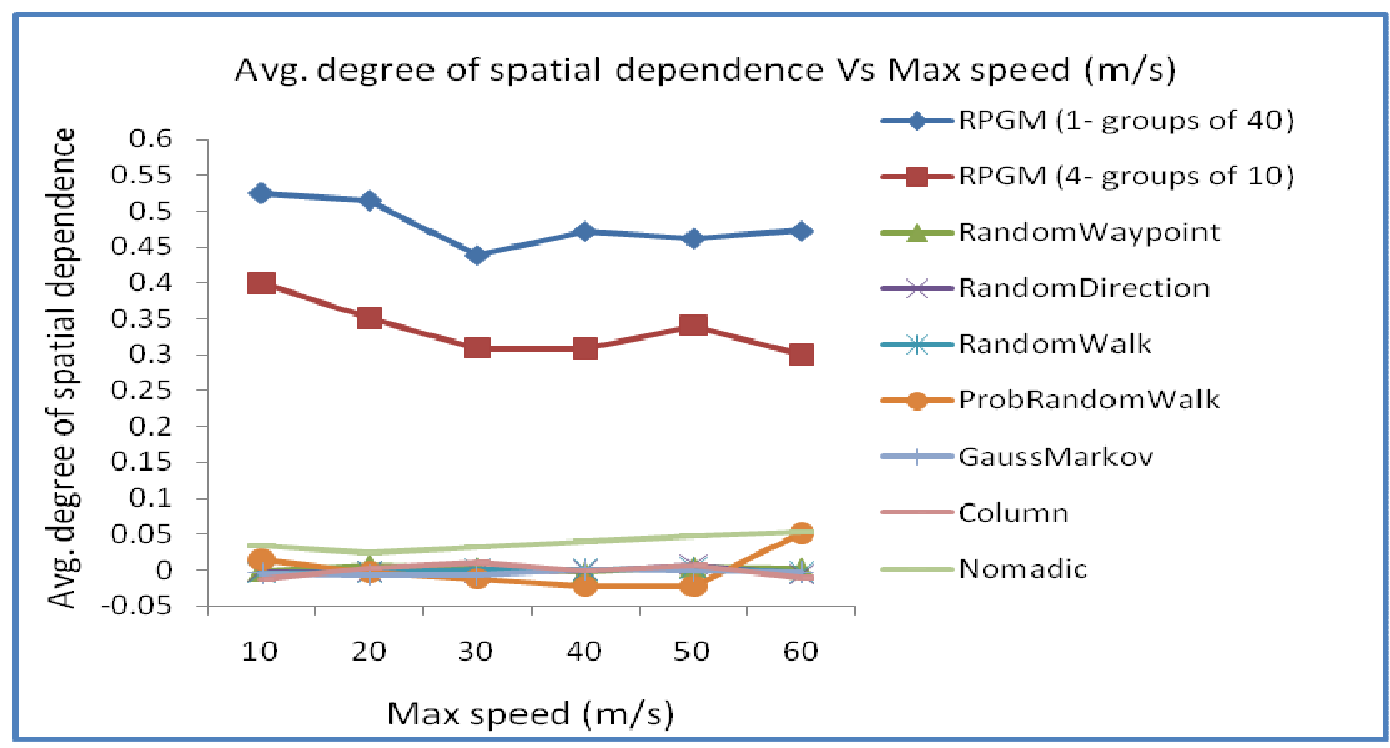

Figure 3 Average degree of spatial dependence Vs Max speed (m/s)

In the case of Random Waypoint and Probabilistic Random Walk it get a very small as the $\mathrm{V}_{\max }$ increase but not consistent, therefore, the simulation validates the rest of mobility models taken in the simulation gone in negative average degree of spatial dependence. In other words, these models are free from spatial dependence and could not be conceded the part of this metrics.

\subsection{Validation of Derived Mobility Metrics}

Average Number of Link Changes: Although the average number link change metric is unable to differentiate the several mobility patterns but in this paper, we made an effort for this task. It has been observed in the simulation the average number of link change probability is very high in case of Original Gauss Markov model as compared to Gauss Markov. The literature [21] study shows the performance of Gauss Markov is better as compared to Original Gauss Markov.

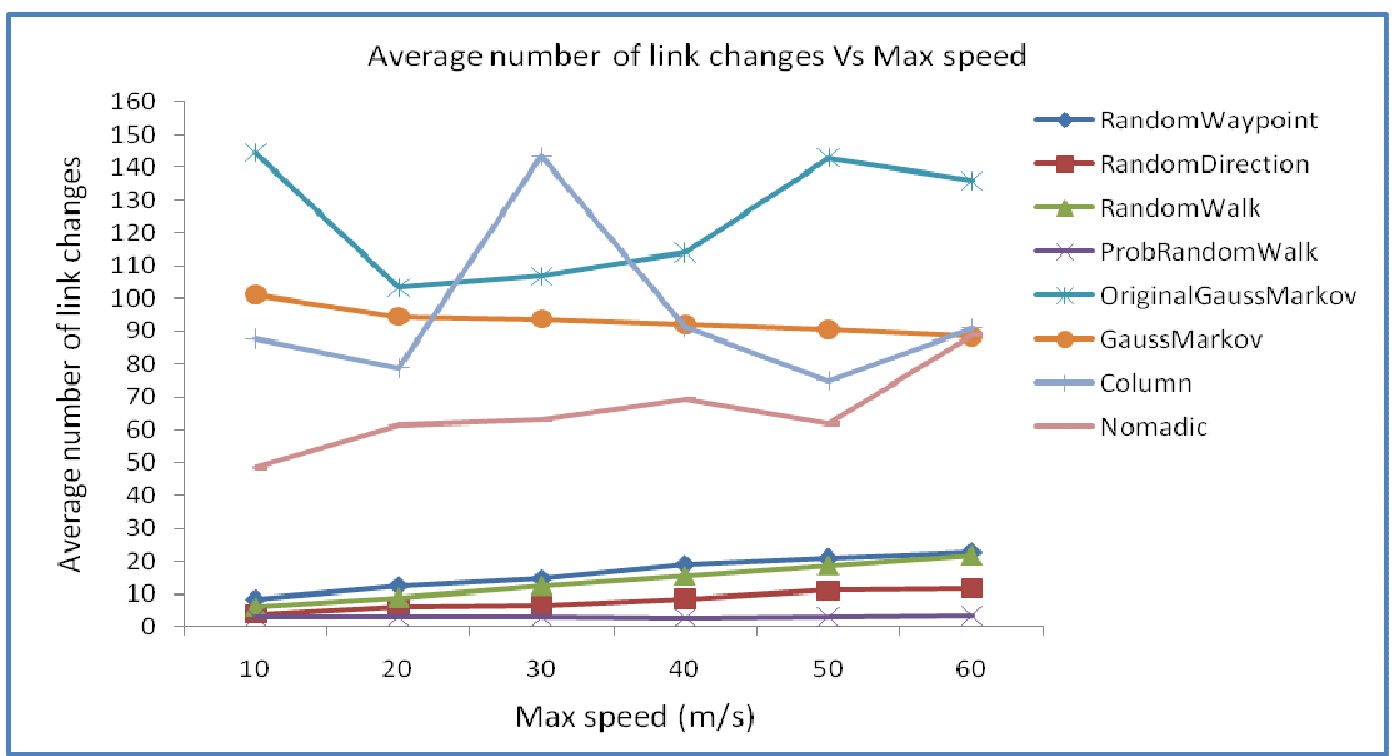

Figure 4 Average number of link changes Vs Max speed (m/s) 
International journal on applications of graph theory in wireless ad hoc networks and sensor networks

(GRAPH-HOC) Vol.3, No.3, September 2011

The average number of link change of Probabilistic Random walk is very less and consistent with $\mathrm{V}_{\max }$ among all simulated mobility pattern. In the case of Random Direction, Random Walk and Random Waypoint, it increases linearly as $\mathrm{V}_{\max }$ increases but these models's link change probability is very less as compared to rest shown in figure 4.

\section{Average Link Duration}

The average link duration as shown in figure 5 has higher and consistent value for RPGM single group than others and in case of Column Mobility Model this value decreases as $V_{\max }$ increases not linearly because of in simulation setup the group change probability and group size standard deviation is zero. This metric value of RPGM (4-group of 10) decreases as $V_{\max }$ increases as comparison to single group because of a single group of 40 nodes breaks into small 4 groups, controlled with its centre head and do not change its membership.

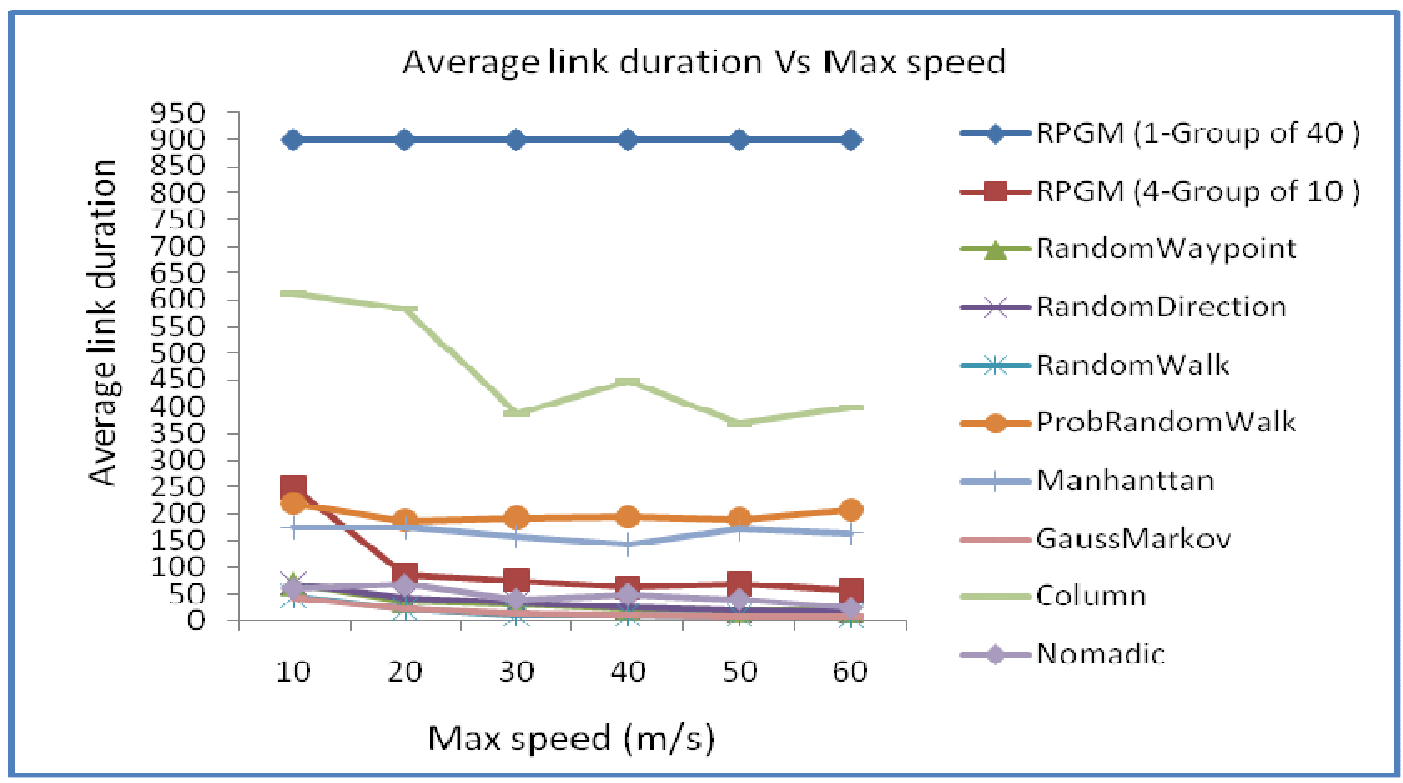

Figure 5 Average link duration Vs Max speed $(\mathrm{m} / \mathrm{s})$

The average link duration of Probabilistic Random Walk model in the beginning and ending of simulation is high but in mid time or when the speed is above $10 \mathrm{~m} / \mathrm{s} \mathrm{\&} 60 \mathrm{~m} / \mathrm{s}$, it is almost consistent. This metric value for Manhattan mobility model decreases as the speed increases and the rest of model has very small value as compared to above described model shown in figure 5 .

\section{Average Path Availability}

It is the fraction of time during which a path is available between two nodes $i$ and $j$. As in figure 6 , the average path availability of Random Waypoint Model has highest value and decreases as the $\mathrm{V}_{\max }$ increases and the Gauss Markov Model average path availability is at second highest position, it decreases as max speed increases. 
International journal on applications of graph theory in wireless ad hoc networks and sensor networks

(GRAPH-HOC) Vol.3, No.3, September 2011

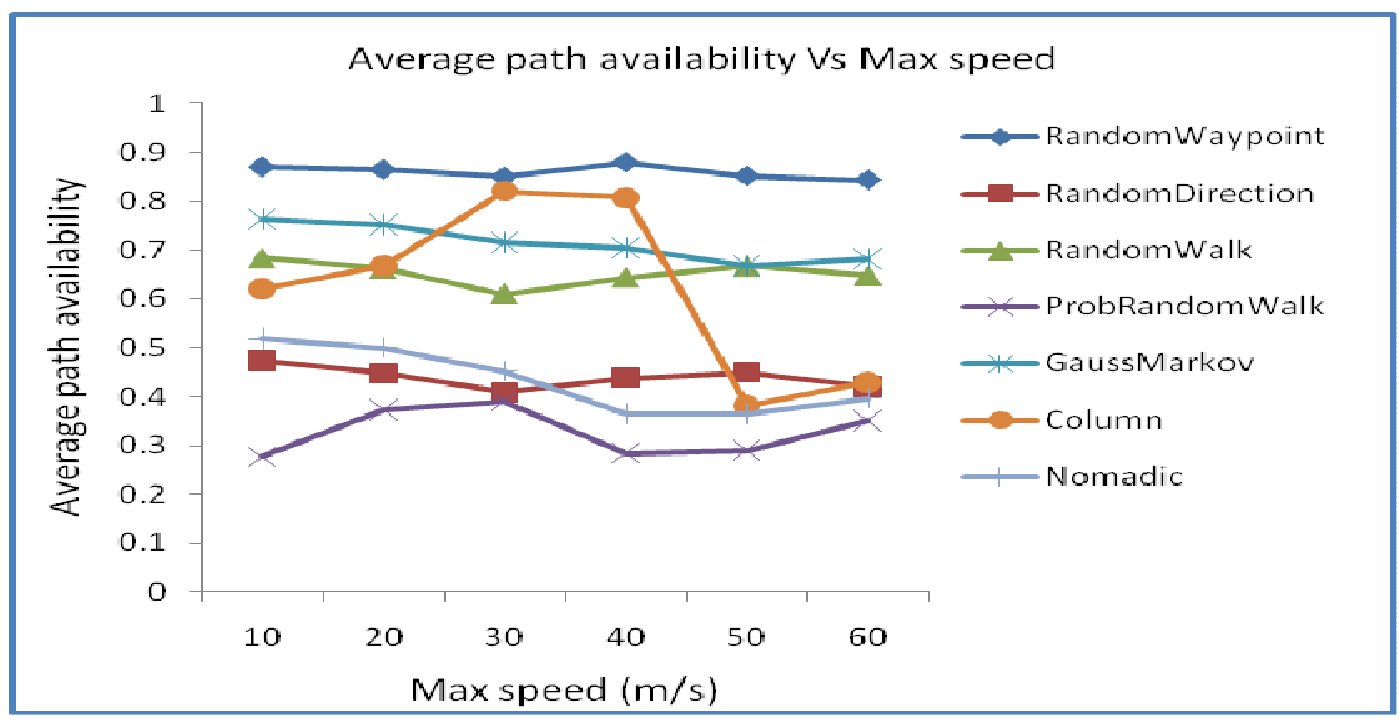

Figure 6 Average link duration Vs Max speed $(\mathrm{m} / \mathrm{s})$

The rest model path availability value is shown in figure 6 .

\section{Total links}

The total links in transmission range 200 meters is carried out for the some mobility model and shown in figure 7. The total links in Gauss Markov is largest as compared to other mobility patterns when the $\mathrm{V}_{\max }$ increases and the total link in Random Walk Model gets its second position of metric value rest of all mobility models. It has approx same values in Manhattan Mobility Model and Column Model as $\mathrm{V}_{\max }$ increases.

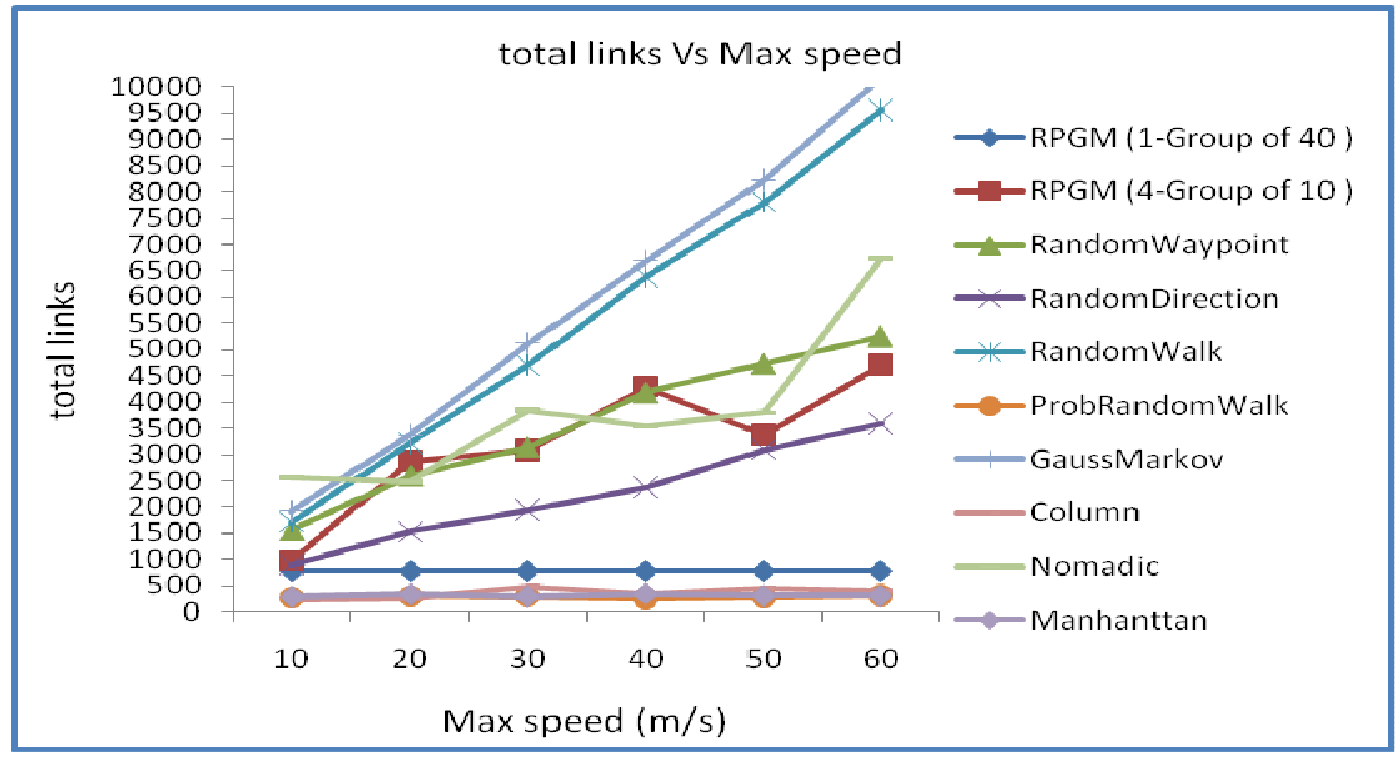

Figure 7 total links Vs Max speed (m/s)

It has approx same values in Manhattan mobility model and column model as $\mathrm{V}_{\max }$ increases. Total links for RPGM (1-Group of 40) is constant while RPGM (4-Groups of 10) increases as $\mathrm{V}_{\max }$ increases. 
International journal on applications of graph theory in wireless ad hoc networks and sensor networks

(GRAPH-HOC) Vol.3, No.3, September 2011

\section{Conclusion and Future Work}

This paper classifies the mobility model by evaluation of mobility metrics i.e direct metrics and derived metrics in figure 1-3, and validates them by consideration of mobility patterns in a systematic fashion. The effect of mobility patterns has been carried out for connectivity graph between the mobile nodes from figure 4-7. Since mobility pattern influences the connectivity graph as shown in simulated results, therefore, the performance of various routing protocol will also affected. Some of the mobility models are classified based on evaluation of these metrics, tabulated in table 1.

Future work is intended to classifying the building blocks and investigating its effect on the performance of various Ad-hoc routing protocols, are mainly based on intuitive analysis.

\section{Acronyms}

$\begin{array}{ll}\text { MNs } & \text { Mobile Nodes } \\ \text { RWP } & \text { Random Waypoint } \\ \text { RWM } & \text { Random Walk Mobility } \\ \text { RDM } & \text { Random Direction Mobility } \\ \text { SR } & \text { Smooth Random } \\ \text { RPGM } & \text { Reference Point Group Mobility } \\ \text { GM } & \text { Gauss Morkov } \\ \text { CMM } & \text { Column Mobility Model } \\ \text { NCMM } & \text { Nomadic Community Mobility Model } \\ \text { SGMM } & \text { Structured- group- mobility model } \\ \text { CVTM } & \text { Complex Vehicular Traffic Models } \\ \text { MGM } & \text { Manhattan Grid Model } \\ \text { RS } & \text { Relative Speed } \\ \text { SR } & \text { Relative Speed } \\ \text { RD } & \text { Relative Direction } \\ \text { LC } & \text { Link Change } \\ \text { LD } & \text { Link Duration } \\ \text { PA } & \text { Path Availability }\end{array}$

\section{Reference}

[1] G.S. Lauer, Packet-radio routing, in: Routing in Communications Networks, ed. M.E. Steenstrup (Prentice Hall, 1995) chapter 11, pp. 351-396.

[2] S. Ramanathan and M.E. Steenstrup, A survey of routing techniques for mobile communications networks, Mobile Networks and Applications 1 (1996) 98-104.

[3] F. Bai, A. Helmy, "A Survey of Mobility Modeling and Analysis in Wireless Adhoc Networks" in Wireless Ad Hoc and Sensor Networks, Kluwer Academic Publishers, 2004.

[4] Nils Aschenbruck, Elmar Gerhards-Padilla, and Peter Martini," A survey on mobility models for performance analysis in tactical mobile networks", JTIT, 2/2008, pp: 54-61.

[5] N. Aschenbruck, E. Gerhards-Padilla, M. Gerharz, M. Frank, and P. Martini, "Modelling mobility in disaster area scenarios", in Proc.10th ACM IEEE Int. Symp. Model. Anal. Simul. Wirel. Mob. Syst.MSWIM, Chania, Greece, 2007.

[6] F. Bai, N. Sadagopan, and A. Helmy, "IMPORTANT: a framework to systematically analyze the impact of mobility on performance of routing protocols for adhoc networks", in Proc. IEEE INFOCOM, San Francisco, USA, 2003, pp. 825-835. 
International journal on applications of graph theory in wireless ad hoc networks and sensor networks

(GRAPH-HOC) Vol.3, No.3, September 2011

[7] Santosh Kumar, S. C. Sharma, Bhupendra Suman,” Mobility Metrics Based Classification \& Analysis of Mobility Model for Tactical Network", International journal of next- generation networks (IJNGN) Vol.2, No.3September 2010, pp.39-51.

[8] C. Bettstetter, G. Resta, and P. Santi, "The node distribution of the random waypoint mobility model for wireless ad hoc networks", IEEE Trans. Mob. Comp., vol. 2, no. 3, pp. 257-269, 2003.

[9] C. Bettstetter and C. Wagner, "The spatial node distribution of the random waypoint mobility model", in Proc. 1st German Worksh. Mob. Ad-Hoc Netw. WMAN'02, Ulm, Germany, 2002, pp. $41-58$.

[10] V. Davies. Evaluating mobility models within an ad hoc network. Master's thesis, Colorado School of Mines, 2000.

[11] C. Chiang. Wireless Network Multicasting. PhD thesis, University of California, Los Angeles, 1998.

[12] E. Royer, P.M. Melliar-Smith, and L. Moser. An analysis of the optimum node density for ad hoc mobile networks. In Proceedings of the IEEE International Conference on Communications (ICC), 2001.

[13] X. Hong, M. Gerla, G. Pei, and C. Chiang. A group mobility model for ad hoc wireless networks. In Proceedings of the ACM International Workshop on Modeling and Simulation of Wireless and Mobile Systems (MSWiM), August 1999.

[14] V. Tolety. Load reduction in ad hoc networks using mobile servers. Master's thesis, Colorado School of Mines, 1999.

[15] M. Sanchez. Mobility models. http://www.disca.upv.es/misan/mobmodel.htm. Accessed on May 13, 2001.

[16] Sanchez and P. Manzoni. A java based simulator for ad-hoc networks. http://www.scs.org/confernc/wmc99/errata/websim/w408/w408.html. Accessed on May 13,2001.

[17] European Telecommunications Standards Institute (ETSI): Universal Mobile Telecom- municatios System (UMTS) - Selection procedures for the choice of radio transmission technologies of the UMTS. UMTS 30.03 version 3.2.0, TR 101 112. 1998.

[18] P. Johansson, T. Larsson, N. Hedman, B. Mielczarek, and M. Degermark. Scenario-based performance analysis of routing protocols for mobile ad-hoc networks. In International Conference on Mobile Computing and Networking (MobiCom'99), pages 195-206, 1999

[19] A. B. McDonald and T. Znati. Link availability models for mobile ad-Hoc networks. Technical Report TR99-07, University of Pittsburgh, Department of Computer Science, May 1999.

[20] "A Mobility Scenario Generation and Analysis Tool”, Version: December 22, 2010, Copyright c 2002-2010 University of Bonn.

[21] B. Liang and Z. Haas. Predictive distance-based mobility management for PCS networks. In Proceedings of the Joint Conference of the IEEE Computer and Communications Societies (INFOCOM), March 1999.

[22] The network simulator - ns-allinone-2.34. 
International journal on applications of graph theory in wireless ad hoc networks and sensor networks

(GRAPH-HOC) Vol.3, No.3, September 2011

Authors

Santosh Kumar (sanjydpt@iitr.ernet.in) received M.Tech. in Computer Science \& Engineering from Aligarh Muslim University, Aligarh (India) in 2007 \& B.E. in Information Technology from C.C.S. University, Meerut (India) in 2003, the member of ACEEE, IAENG, ISOC (USA), Reviewer of Active International Journal "IJACSA", has published over seven research papers in National \& International Journals/conferences in field of Wireless Communication Networks, Mobile Computing and Grid Computing. He has more than 4 years of experience in teaching of UG (B.Tech.) \& PG (M.Tech.) level courses in teaching as a Lecturer/Assistant Professor. Currently, he is a Doctoral candidate in Indian Institute of Technology, Roorkee (India) in the field of Wireless Communication Networks since august 2008.

Dr. S.C. Sharma (scs60fpt@iitr.ernet.in) received M.Tech. (Electronics \& Communication Engg. and Ph.D. Electronics \& Computer Engg.) in 1983 and 1992 respectively from IIT Roorkee (erstwhile University of Roorkee), the honorary member of NSBE, ISOC, and IAENG, ACEEE. He started his career as R \& D Engineer in 1983 then joined teaching profession in Jan. 1984 in IIT-Roorkee and continuing till date. He has published over hundred thirty six research papers in national and international journals/conferences and supervised more than 30 projects/dissertation of PG students. He has supervised several Ph.D. in the area of Computer Networking, Wireless Network, Computer Communication and continuing supervising Ph.D. in the

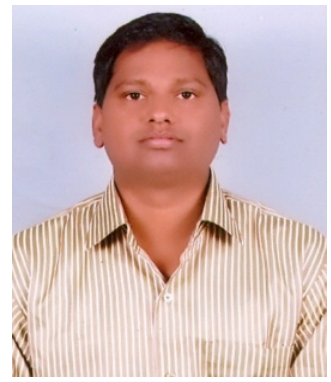
same area. Currently, he is supervising Six Ph.D. Scholars. He has successfully completed several major research projects independently and many minor research projects related to Communication and SAW filter Design sponsored by Government of India. IIT-Roorkee has awarded him the Khosla annual research prize with best research paper in the year 2000. His many research papers have been awarded by National and International Committees. He has worked as research scientist at FMH, Munchen, Germany in the year 1999. He is the active reviewer of two reputed International Journals and Editor of National Journal (BITS, PILANI). He has also worked as Group leader of Electronics \& Instrumentation Engg. Department of BITS-Pilani-Dubai Campus, from Aug. 2003 to Aug. 2005. Presently he is continuing as Associate Professor at IIT Roorkee-India.

BhupendraSuman (bhupendra.suman@ rediffmail.com) received his B.E (Electronics \& Communication Engineering) from MANIT Bhopal, India in 2000 and joined DRDO in Defence Electronics Application Laboratory(DEAL) Dehradun. In DEAL he has worked in various communication projects including Combat Net Radio (CNR). He was awarded Laboratory Scientist of the year 2005 for his contribution in project. Presently he is working in the field of tactical MANET as a Doctoral candidate at IIT Roorkee-India since January 2009.

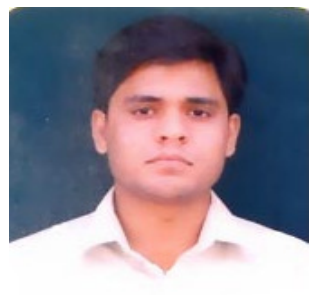

